•综述・

\author{
声景生态学研究进展和展望 \\ 赵 莹 1,2 申小莉 ${ }^{*}$ 李 是 ${ }^{3}$ 张雁云 ${ }^{4}$ 彭任华 5 马克平 ${ }^{1}$ \\ 1 (中国科学院植物研究所植被与环境变化国家重点实验室, 北京 100093) \\ 2 (中国科学院大学, 北京 100049) \\ 3 (北京大学生命科学学院, 北京 100871) \\ 4 (北京师范大学生命科学学院, 北京 100875) \\ 5 (中国科学院声学研究所噪声与振动重点实验室, 北京 100190)
}

摘要: 声景生态学以景观中的声音为研究对象, 探讨其在不同时空维度上的分布和变化模式, 从而揭示自然环 境、野生动物和人类活动的相互作用关系。本文通过系统检索声景生态学研究的相关文献, 回顾了该学科的研究 框架和研究方法, 总结了目前常用的声学指标, 重点归纳了声景生态学的研究内容, 包括声景组成和各组分间的 相互作用, 声景的时空格局, 以及声景生态学在生物多样性监测中的应用。目前, 声景监测中存在的问题主要包括 监测的生态系统类型和物种类群有限、声学指标效力有待提高等。建议未来着重推进建立系统性的声景监测网络 和数据管理平台, 开发和完善音频数据采集、分析方法和评估指标, 并重视声景数据的采集, 将声景视作一种资源 进行研究和保护。

关键词: 声景监测; 声学指标; 声学生态位; 生物多样性监测; 自动录音技术

\title{
Progress and outlook for soundscape ecology
}

\author{
Ying Zhao ${ }^{1,2}$, Xiaoli Shen ${ }^{1 *}$, Sheng $\mathrm{Li}^{3}$, Yanyun Zhang ${ }^{4}$, Renhua Peng ${ }^{5}$, Keping $\mathrm{Ma}^{1}$ \\ 1 State Key Laboratory of Vegetation and Environmental Change, Institute of Botany, Chinese Academy of Sciences, \\ Beijing 100093 \\ 2 University of Chinese Academy of Sciences, Beijing 100049 \\ 3 School of Life Sciences, Peking University, Beijing 100871 \\ 4 College of Life Sciences, Beijing Normal University, Beijing 100875 \\ 5 Key Laboratory of Noise and Vibration Research, Institute of Acoustics, Chinese Academy of Sciences, Beijing 100190
}

\begin{abstract}
Soundscape ecology studies sound patterns across different spatial and temporal scales, which reflects coupled natural-human dynamics in a changing landscape. Here, we synthesized a list of peer-reviewed studies on soundscape ecology to summarize the conceptual framework of the field, the methodology used, and acoustic indices developed from these studies. Studies on soundscape ecology are currently focused on the following aspects: (1) acoustic composition of soundscape; (2) acoustic interactions between soundscape components; (3) temporal patterns and spatial variability in the soundscapes; (4) the development of acoustic indices for biodiversity monitoring. We suggest the following as focuses for future studies in soundscape ecology and biodiversity conservation: (1) establishing a systematic acoustic monitoring network and data management platform; (2) developing new methods for data collection and analysis (e.g., recording matrix and machine learning algorithm); and (3) treating soundscape ecology as an important resource for future research and conservation.
\end{abstract}

Key words: soundscape monitoring; acoustic indices; acoustic niche; biodiversity monitoring; automated acoustic recording

“声景”(soundscape)一词由Southworth (1967)提出, 指景观中全部声音信息的总和, 在城市规划

收稿日期: 2020-08-11; 接受日期: 2020-08-28

基金项目: 美丽中国生态文明建设科技工程专项(XDA23080302)

* 通讯作者 Author for correspondence. E-mail: xlshen@ibcas.ac.cn 
(Southworth, 1967)、建筑设计(Thompson, 2004)、音 乐作曲(Truax，2008)等领域均有应用。20世纪60年 代末, 加拿大作曲家Raymond Murray Schafer主持 实施了首个声景研究项目 “The World Soundscape Project”, 并创立了声学生态学(acoustic ecology), 呼吁人们关注环境中声音及其与人类活动的相互 作用关系(Schafer, 1969, 1970)。1987年, 美国音乐学 家和声景生态学家Bernie Krause (1987)提出了“声 学栖息地理论” (acoustical-habitat theory), 认为每 个生态系统有其独特的、反映其内部特征的生物声 学谱(acoustic bio-spectrum)。2011年, 美国普渡大学 的Bryan Pijanowski和意大利乌尔比诺大学的Almo Farina将声学生态学与景观生态学的理论框架结合, 主张研究声景的时空分布模式和生态过程 (Pijanowski et al, 2011b), 并在此研究框架和研究内 容的基础上提出了一个新的学科方向一一声景生 态学 (soundscape ecology), 也有学者称其为 “ecoacoustic” (Farina \& Gage, 2017)。

Pijanowski等(2011b)沿用从声音来源角度描述 声景构成的方法(Krause, 1987), 将声景分为3个组 成部分：生物声音 (biophony), 物理环境声音 (geophony, 如风声、雨声、流水声), 以及人造声音 (anthrophony)。声景生态学研究声景在不同时空维 度上的分布和变化模式, 及其与生物和非生物环境 之间的关系, 以此揭示其所反映的人类活动与生态 过程(Pijanowski et al, 2011b)。Pijanowski等(2011a) 详细阐述了自然和人为因素改变声景格局的过程 和机理: 大气动力学改变自然环境, 进而影响动物 的生活史和栖息地, 以及生物多样性，同时影响地 形、光周期等地球物理特征。人类通过建筑环境和 土地改造与大气过程共同影响声景特征, 具体体现 在4个方面: (1)声学组成(指声景的频段和振幅大小); (2)声景的时间格局; (3)声景的空间变异性; (4)在自 然和人为因素介导下, 生物声音、物理环境声音和 人造声音间的交互作用。大气动力学和人为活动可 以改变声景特性, 同时被改变的声景将反作用于生 物和人类系统, 影响动物的种群和群落动态(Barber et al, 2010), 以及人类的健康和对环境的感知。

随着声景生态学概念的传播, 越来越多的研究 者认识到声景在生态系统中具有重要的生态功能 (Farina \& Gage, 2017), 与声景相关的研究逐步深入 开展。我们在Web of Science (www.webofknowledge. com)上检索“soundscape ecology”或“ecoacoustic*”, 截至2019年12月, 共获得168篇声景生态学研究论 文，相关研究自2014年起快速增加，2014年之后发 表的相关论文占该领域论文总数的 $93.4 \%$ 。

本文介绍了声景生态学的研究框架、研究方法 和研究内容, 总结了目前常用的声景评估指标, 分 析了已有研究的局限性, 并对未来声景生态学发展 方向提出了建议, 以期为此领域研究的开展提供思 路和参考。

\section{声景生态学研究方法}

\section{1 数据采集方法}

声景生态学主要利用自动录音机 (automated acoustic recorders)采集音频数据。近年来, 可编程自 动录音机的出现促进了声景生态学及其他与声学 监测相关学科的快速发展(Sugai et al, 2019)。动 录 音机利用声学传感器(水听器hydrophone或麦克风 microphone)采集环境中的声信号, 其中水听器适用 于接收水中的声信号，麦克风适用于接收陆地中的 声信号。声学传感器将声波转化为电信号(Drafts, 2001), 再通过脉冲编码调制法 (pulse code modulation, PCM) 将电信号量化, 转换为数字信号 储存到存储介质中。自动录音机可按照用户设定的 日程，在固定的时间自动进行声音采集。近年来电 池寿命的延长、内存介质容量的增大等技术上的进 步，提高了录音设备的野外工作时长，满足了声景 生态学大尺度、长时期、自动化的声学监测需求。

根据人类听觉的频带范围, 声音信号可以划分 为可听声频带(20 Hz-20 kHz)、超声频带(> $20 \mathrm{kHz})$ 以及次声频带 $(<20 \mathrm{~Hz})$ 。对于可听声频带的声音, 研究人员使用普通录音机录制即可。某些动物(例如 蝙蝠、某些鲸豚类物种)具有特殊的超声回波定位能 力, 会发出超声波, 对这些动物可以通过以较高采 样频率(高达400 kHz)录制音频的方法，探测并记录 环境中它们发出的超声波(Fenton \& Bell，1979; Russ, 1999)。此外，亚洲象(Elephas maximus)和非洲 象(Loxodonta africana)会发出次声波进行远距离家 族间的联络(Payne et al, 1986; Venter \& Hanekom, 2010)。一般使用次声波探测器探测次声波, 主要应 用于各类地质灾害监测中(吕君等, 2012), 在声景生 态学研究中应用较少。

目前常用的自动录音设备有用于监测陆生动 
物的Song Meter (Wildlife Acoustics Inc., Maynard, MA, USA)、ARBIMON (ARBIMON/Sieve Analytics, Puerto Rico)和BATLOGGER (Elekon, Switzerland), 用于监测海洋哺乳动物的C-POD (Chelonia Limited, Cornwall, UK)等。部分设备结合了新一代传输技术 可实时回传数据, 比如FrogPhone (Garrido et al, 2020)、Robust (Sethi et al, 2018)。近年来也出现了 外形更小巧、价格更低廉的迷你自动录音设备, 如 AudioMoth (Hill et al, 2018)、 Solo (Whytock \& Christie, 2017)、Song Meter Mini (Wildlife Acoustics Inc., Maynard, MA, USA)等。

自动录音设备通常被布设在野外调查点, 按照 预先设定的启动时间和录音时长录制声音。当研究 对象是明确的某个动物类群时, 需要有针对性地选 择录音位点, 比如以森林鸟类为研究对象时, 可将 录音设备布设在离地约 $1.5 \mathrm{~m}$ 的树干上, 并回避环 境声音(如湍急溪流声、录音设备周围树枝摇摆的声 音)的干扰。建议在采集录音的同时记录调查日的天 气状况, 可在后期数据处理时, 根据需要识别和去 除带有较大环境背景噪声(如雨声、风声)的录音, 从 源头上削弱噪声对后续音频数据分析处理的影响。

\section{2 数据分析处理方法}

声波在被声传感器转换为电信号之后, 通过模 数转换(analog to digital, AD) 芯片转换为数字信号, 一般以时间-幅值形式呈现(图1A), 其中横轴为时
间, 纵轴为幅值。通过短时快速傅里叶变换(short time fast fourier transform, STFT), 原始声音的时域 信号被转换为具有随时间变化的不同频率、振幅和 相位的正弦波信号的叠加，构造时间、频率以及振 幅这三种变量的三维图就可以得到声谱图 (spectrogram)(图1B)。在声谱图中, 横坐标表示时间, 纵坐标表示频率，不同颜色深度表示振幅大小。声 谱图能够清晰地反映频率、振幅随时间的变化关系, 是声景分析中的重要手段。

针对动物类群, 研究者通常希望鉴定出录音数 据中的发声物种。传统的方法需要手动扫描声谱图, 人为判读录音中的目标声音(Wimmer et al, 2013), 十分耗时。目前, Kaleidoscope, ARBIMON II, Raven 等软件嵌入了基于机器学习算法的自动识别技术, 能够极大地减少扫描音频数据集时所用的时间，快 速地检测出发声物种(Brandes, 2008)。但目 前, 自动 识别结果的准确性仍存在假阳性和假阴性比例较 高的问题(Swiston \& Mennill, 2009; Towsey et al, 2012)。

与传统的录音数据处理方法不同, 声景生态学 关注的重点通常不是录音里的物种，而是用于录音 数据的音频频域和时域的特征。这些特征可以使用 声景指数(即声学指标)来描述，比如多样性或复杂 度等(Sueur et al, 2008, 2014)。声学指标可以分为两 大类：(1) $\alpha$ 声学指标：用于评估声景或动物群落的
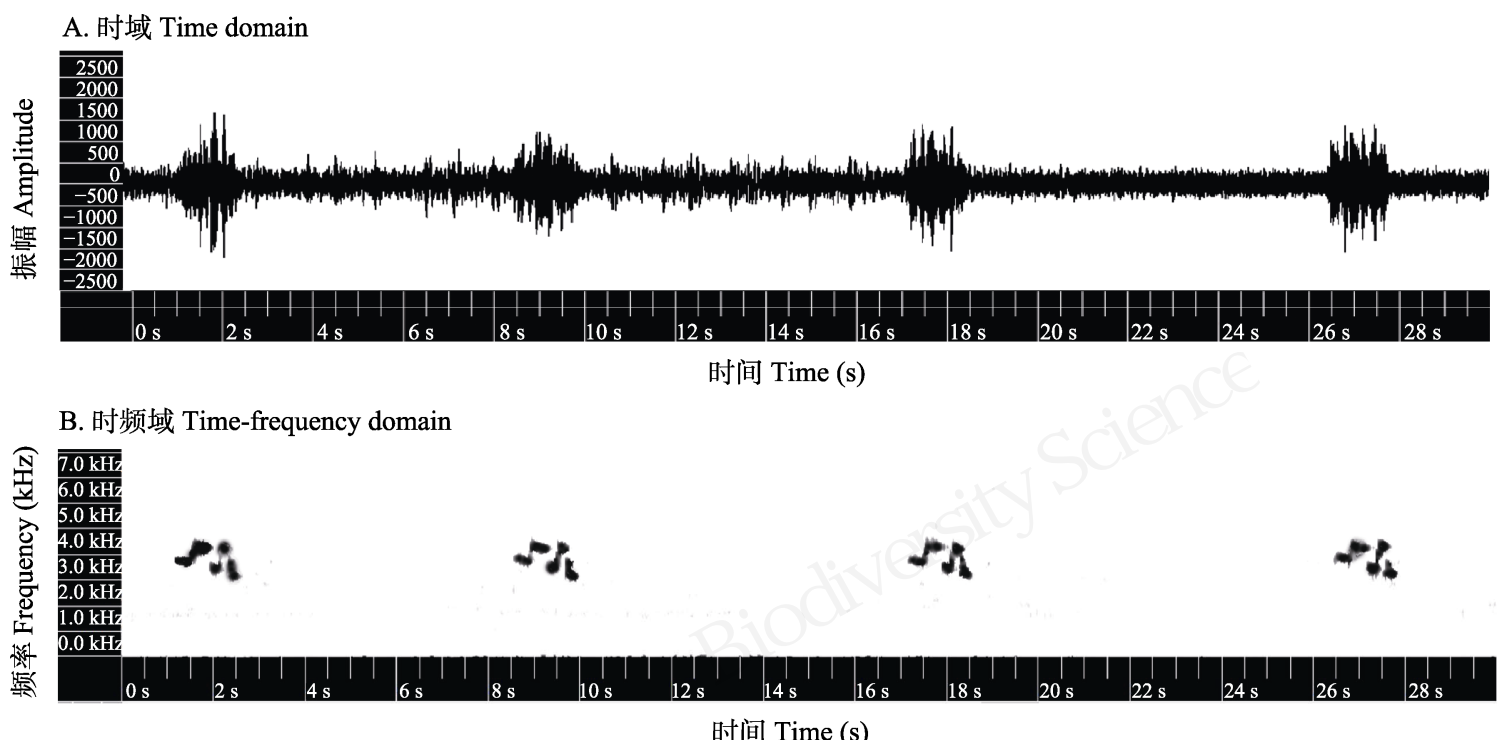

图1 在Kaleidoscope pro5软件中显示的褐顶雀䴗(Alcippe brunnea)鸣唱的波形图(A)和声谱图(B)

Fig. 1 Oscillogram (A) and spectrogram (B) of the song of Alcippe brunnea shown in Kaleidoscope pro5 (Wildlife Acoustics Inc., Maynard, MA, USA) 
声音多样性或复杂性; (2) $\beta$ 声学指标: 用于评估不 同动物群落间或同一群落不同时段间的声音多样 性的差异。Buxton等(2018b)分析了 71篇与声学指标 有关的文献, 统计了用于不同目的的 60 余种声学指 标。这些指标大部分可在R语言环境中(比如软件包 tuneR, seewave, soundecology)实现分析。Box 1列举 了研究中较常使用的10个声学指标及其定义和相 关研究。

声学指标评价生物多样性的效果受诸多因素
影响, 其中，背景噪声，如风声、雨声、人为制造的 声音均是重要的影响因素。地震勘测活动可能掩盖 声学熵指数(acoustic entropy index, $H$ )与鲸鱼发声 频率的线性关系(Parks et al, 2014); 昆虫鸣声、环境 背景音、人为噪声数量的增加, 可能增加基于声学 指标预测脊椎动物多样性水平的模型的残差 (Buxton et al, 2018b)。后期实验室分析时，通过噪声 补偿(Parks et al, 2014)、使用合适滤波器(Farina \& Pieretti，2014)等信号处理方式，可以达到降噪的目

声音多样性指数 Acoustic diversity index (ADI) (Villanueva-Rivera et al, 2011)

提取每一频段中超过特定声压级阈值(默认为 $-50 \mathrm{dBFS}$ )的声信号参数, 计算Shannon指数 (Shannon's index), 指示声音多样性: $A D I=\sum_{i=1}^{s} p_{\mathrm{i}} \ln p_{\mathrm{i}}, p_{\mathrm{i}}$ 是声信号在第 $\mathrm{i}$ 个频段所占比例, $s$ 是频 段数量。Villanueva-Rivera等(2011)应用 $A D I$ 比较了不同地点间以及一天中不同时段间的声景差 异。

声音均匀度指数 Acoustic evenness index (AEI) (Villanueva-Rivera et al, 2011)

提取每一频段中超过特定声压级阈值(默认为 $-50 \mathrm{dBFS}$ )的声信号参数, 计算吉尼指数(Gini index) 来表示声信号强度在不同频段的不均等程度。Villanueva-Rivera等(2011)用AEI比较不同土地利用 类型录制的音频信号在频谱上的差异性。

人类产生声音(anthropophony)与生物产生声音(biophony)声学成分间的比率, 评估人为千扰对声 标准化声景差异指数 Normalised 景的影响程度。Kasten等(2012)在建立远程声学评价实验室时, 利用NDSI篎选录音。 difference soundscape index (NDSI) (Kasten et al, 2012)

$\beta$ 声学指标 $\beta$ acoustic index 声学差异性指数 Acoustic dissimilarity index (D) (Sueur et al, 2008)

时域差异性指数(temporal dissimilarity index, $D_{\mathrm{t}}$ )和频域差异性指数(spectral dissimilarity index, $D_{\mathrm{f}}$ ) 的乘积, 评估群落间声信号在时域和频域上的差异性: $D_{\mathrm{t}}=0.5 \times \sum_{t=1}^{N}\left|A_{1}(t)-A_{2}(t)\right|$,

$D_{f}=0.5 \times \sum_{t=1}^{n}\left|S_{1}(f)-S_{2}(f)\right|, D=D_{t} \times D_{f}, D \in[0,1]$

Sueur等(2008)用 D比较坦桑尼亚干旱低地沿海破碎化森林和完整森林动物发声活动的差异。
} 
的。如果无法去除录音中的噪声, 可以通过统计学 手段屏蔽噪声的影响, 比如将信噪比(signal-noise ratio, SNR) 设为控制变量进行偏相关分析 (Desjonqueres et al, 2015)。此外, 声学指标的效果还 受到目标鸣声的类型、鸣声间的重叠程度、发声强 度、发声频次以及傅里叶变换长度(FFT size)大小的 影响(Gasc et al, 2015; Zhao et al, 2019)。如Zhao等 (2019)通过模拟实验发现, 声学群落中发声频率的 下降, 会使得声音多样性指数 $(A D I)$ 、声音均匀度指 数 $(A E I)$ 、声学熵指数 $(H)$ 等指标的标准差增大。 Harris等(2016)用声学指标评估温带珊瑚礁鱼类多 样性时, 发现傅里叶变换长度从512上升至1,024后, 声学熵指数 $(H)$ 数值显著增加。

\section{2 声景生态学的主要研究内容}

声景生态学早期研究集中于描述陆地生态系 统中的声景在时间尺度上的动态变化以及在环境 梯度上的空间异质性(Krause et al, 2011), 并开发了 一系列声学指标 (Sueur et al, 2008; VillanuevaRivera et al, 2011)及音频数据分析平台(Kasten et al, 2012)用于野生动物研究(Lillis et al, 2014; Buxton et al, 2016)、栖息地环境质量评价(Gomez et al, 2018)、 生物多样性评估(Pieretti et al, 2011; Gasc et al, 2013a)、保护成效评估(Bobryk et al, 2016)以及气候 变化和人类开发建设活动对生物多样性影响的调 查(Krause \& Farina, 2016; Deichmann et al, 2017; Burivalova et al, 2018; Gasc et al, 2018)等领域。随后, 声景生态学研究范围从陆地生态系统扩展到淡水 和海洋生态系统(Harris et al, 2016; Putland et al, 2017), 研究的动物类群从鸟类扩展到鱼类(Parsons et al, 2016)、珊瑚虫(Lillis et al, 2014, 2016)、鸣虫 (Roca \& Proulx, 2016)等, 研究的对象也从单一的物 种类群扩展到多物种类群(Ferreira et al, 2018)。

\section{1 声景的组成及有效传播}

同域生活的动物发出的声音具有多样化的频 段范围和特征(图2)。在同一时间、同一地点, 不同 频率和振幅的声音共同构成了声景, 即声景的声学 组成(acoustic composition) (Pijanowski et al, 2011a)。 研究者提出了相关假说试图解释动物声音多样性 的成因, 如声学生态位假说 (acoustic niche hypothesis, Krause, 1993)和声学适应假说(acoustic adaptation hypothesis, Morton, 1975)。

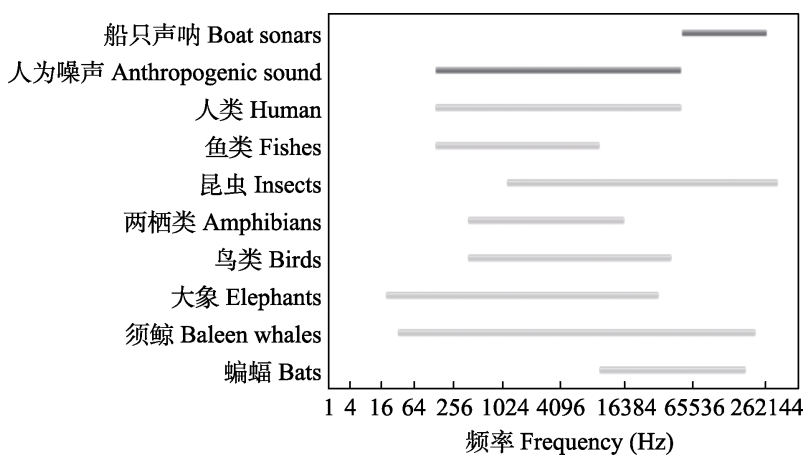

图2 常见人为噪声源与动物发声的频率范围

Fig. 2 The frequency range of common anthrophonic noise and animal vocalization

声学生态位假说认为, 物种间的竞争排斥作用 会促进不同物种发出的声音在时间和频率上出现 分化，以减少种间声信号的重叠，实现对声学空间 的高效利用(Krause，1993)。已有研究发现，鱼类 (Ruppé et al, 2015)、两栖类 (Villanueva-Rivera, 2014)、鸟类(Halfwerk \& Slabbekoorn, 2009)等动物 发出的声音存在种间频率分化。比如，在南非东南 部海域索德瓦纳湾中，日间和夜间发声的鱼类类群 在组成上有所差异，声音在时间上存在分化(Ruppé et al，2015)。在波多黎各高地中，卵齿蟾属 (Eleutherodactylus)的不同物种发出的声音占据不同 频段(Villanueva-Rivera, 2014)。

声学适应假说认为, 环境倾向于篮选和保留传 播距离远且保真度损失小的声信号, 因此动物发出 的声音会根据其所处环境做出适应性改变，以提高 声信号传输的效率(Morton，1975)。Velásquez等 (2018)在南美洲 8 个不同地点收集了南美细趾蟾 (Pleurodema thaul)的叫声, 并在这 8 个地点分别做 声信号传播试验，结果显示叫声在原发地的传播效 率最高。声学适应性假说在鸟类和哺乳动物的研究 中也得到过验证(Ey \& Fischer, 2009)。除此之外，环 境过滤作用可能去除部分声信号，也会导致不同栖 息地的声景呈现出相异的声学特征。

气候变化正在改变声景的组成。气候变化影响 声信号在介质中传递的衰减速率，进而引起生物发 声行为的改变。例如，当气温升高时，高频超声波 信号的衰减速率增加，在蝙蝠捕食过程中的使用频 率有降低的趋势(Luo et al, 2014)。空气湿度上升会 造成大气对声音吸收的增加(Snell-Rood, 2012), 因 此，在高湿环境中，北美的林莺科鸟类物种会降低 
叫声的频率带宽和鸣叫持续时长。在海洋环境中, 海水酸化减少了海水对低频声信号的吸收, 海洋动 物因此改变发声频率、持续时长和声强, 以避免自 己发出的声信号被低频噪声掩盖(Brewer \& Hester, 2009)。

\section{2 声景组成成分间的相互作用}

声景的3个组分(生物声音、物理环境声音和人 造声音)间存在相互作用(Pijanowski et al, 2011a)。生 物的发声行为会受到物理环境声音的影响。例如, 动物通常在刮风或下大雨时停止发声(Feng \& Schul, 2007), 或者倾向于在一天中风速最低时(例如黎明 和黄昏)发出和鸣 (Cuthill \& Macdonald，1990; Hutchinson, 2002; Berg et al, 2006; Hardouin et al, 2008)。为了实现有效通讯, 动物发出声音的频率往 往高于背景风声的频率(Greenfield, 1994); 如帝企 我鸟(Atenodytes patagonicus)在强风噪声下, 通过提 高呼叫频率和单次呼叫的音节数来保持通讯效率 (Lengagne et al, 1999)。

近年来, 人造声音如何影响生物的发声成为声 景生态学的研究热点之一。人造声音来源众多, 包 括工业、交通、人类的生活娱乐等诸多方面。一些 人造声音与动物发出的声信号在频率上重叠(图2), 可能干扰两栖类、鸟类和哺乳动物的种内通讯, 进 而危害动物繁殖(Smott et $\mathrm{al}, 2018$ )、栖息地选择 (Lecchini et al, 2018)、捕食者识别(Ferrari et al, 2018) 等重要行为。有研究表明即使人为噪声与水鼠耳蝠 (Myotis daubentoniid)的发声在频率上不重叠, 也会 显著降低其受食效率(Luo et al, 2015b)。噪声环境中 生活的动物在发声上会做出适应性改变(如调整发 声时长、频率或振幅), 以提高声信号的传输效率, 即“隆巴德效应(Lombard effect)” (Lombard, 1911)。

例如, 长期在嘈杂环境中生活的鸟类通过提前和鸣 时间、增加鸣唱时长(Sierro et al, 2017)、提高鸣唱 频率(Slabbekoorn \& Peet, 2003)、增大鸣唱振幅 (Cynx et al, 1998; Brumm, 2004)等途径, 提高个体 间的通讯效率。蝙蝠用增大发声振幅和频率、延长 发声时长、增加信号冗余、降低发声复杂程度等方 式, 来应对噪声对蝙蝠个体之间通讯的干扰(Hage et al, 2013; Luo et al, 2015a; Jiang et al, 2019)。

\section{3 声景的时空格局}

声景具有典型的时间格局, 在温带森林 (Depraetere et al, 2012)、热带雨林(Rodriguez et al,
2014)、城市(Botteldooren et al, 2006)、河流(Martin \& Popper, 2016)、湖泊(Gage \& Axel, 2014)、海洋 (Staaterman et al, 2014)等生态系统中，均存在日、月 或季节性的变化规律。声景的时间模式与生物的活 动节律和生命周期有着密不可分的联系, 又被称为 “自然的节奏”(rhythms of nature)。例如，鸟类的晨昏 和鸣行为使得晨间和傍晚的声学丰富度指数 $(A R)$ 显 著高于一天中的其他时段(Depraetere et al，2012; Gage \& Axel, 2014; Rodriguez et al, 2014)。在特定季 节里，昆虫和两栖类动物常会出现夜间发声活跃的 现象(Rodriguez et al, 2014)。在每 洋中, 海胆(Putland et al, 2017)、虾类(Lillis \& Mooney, 2018)、鱼类(Rice et al, 2017)、鲸豚类(Au et al, 2000; Guan et al, 2016) 均有和鸣现象，并存在昼夜、季节性的规律，同时 受到天体运动和环境的影响 (Lillis \& Mooney, 2018)。例如, 珊瑚礁声景存在月节律, 在新月时增 强，满月时减弱(Staaterman et al, 2014); 温带沿海 声景的夏季声强高于冬季(Radford et al, 2008); 在 开阔海域中, 大型海洋哺乳动物影响季节性声音强 度, 例如座头鲸(Megaptera novaeangliae)在冬春之 交繁殖季节发出的声音, 会增加海洋声景中1002,000 Hz频率段的声强(Bittencourt et al, 2016); 而 在城市和农村生态系统中, 声景的时间变化模式主 要受人类活动驱动(Liu et al, 2013)。

受自然因素(纬度、海拔、植被类型、植被垂直 结构等)和人为因素(土地利用类型、人为噪声等)的 影响，声景呈现出空间异质性。由于声景与发声动 物的分布紧密相关, 因而在较大的空间尺度上，可 能呈现出沿纬度梯度和海拔梯度的分布格局。比如, 在赤道地区较高的生物多样性环境里, 声景的多样 性和复杂性也可能更高(Pijanowski et al, 2011a)。与 低海拔的常绿阔叶林相比, 高海拔针叶林中声音多 样性指数 $(A D I)$ 较低, 但季节性变化更为明显(Lin et al, 2017)。声景在不同的植被类型和植被垂直结构 中存在差异。蔡学林等(2010)依据声学特征的差异, 建立了不同类型的森林声景资源信息库(包括阔叶 林声景、针叶林声景、毛竹林声景、灌木林声景、 小溪声景、河流声景、瀑布声景7种类型); Rodriguez 等(2014)发现法属圭亚那热带森林中林冠层声音的 振幅高于林下层; Lin等(2017)的研究结果显示常绿 阔叶林中的生物声音多样性要高于针叶林。 
近年来, 气候变化正在改变物种分布格局和物 候特征, 进而改变声景的时空格局。Parmesan (2006) 综述了过去几十年来全球变暖引起的物候变化, 发 现许多生物(特别是两栖动物)的物候受到了温度升 高的明显影响。例如, 相比于1900年, 纽约伊萨卡 蛙类繁殖和鸣的时间提前了 10-13天 (Gibbs \& Breisch， 2001); 北美繁殖鸟类灰胸丛鸦 (Aphelocoma ultramarina)的繁殖期提前(Brown et al, 1999)。在北美, 由于气候变暖, 外来种红嘴相思鸟 (Leiothrix lutea)的分布范围逐步扩大, 与同域分布 的黑顶林莺(Sylvia atricapilla)和其他鸣禽对声学空 间的竞争更为激烈(Farina et al, 2013)。

相比生物声音, 人造声音的频率一般较低, 但 强度较高、持续时间长(Dooley \& Brown, 2019)。人 造声音可以直接塑造声景格局, 进而影响声景环境 中的野生动物。研究发现城市化过程中, 声景中人 造声音的比例增加, 生物声音的比例减少(Kuehne et al, 2013)。在海洋环境中, 珊瑚礁鱼类在幼年阶段 通过感知声环境选择合适栖息地, 船只噪声会掩蔽 声环境, 干扰鱼类在适宜生境定居(Holles et al, 2013)。同时, 人类活动也可以通过改变物种及其栖 息地的空间分布格局, 间接地改变声景的空间格 局。例如, 被人类开发利用后的森林(如开唇种植棕 榈林后) 里, 声景在晨昏时段的饱和度下降 (Burivalova et al, 2018), 在空间上的同质性增加 (Burivalova et al, 2019)。森林破碎化会改变森林斑 块大小和联通性, 进而改变生物声音和人造声音的 功率水平(Tucker et al, 2014)。声景的声学特征与土 地利用类型和距交通密集区的距离紧密相关, 在城 市到乡村的梯度下, 生物声音的功率谱密度(power spectral density)上升, 人造声音的功率谱密度下降 (Joo et al, 2011)。因此, 一些能反映人类活动干扰强 度的声学指标可用于评估生态系统质量(Fuller et al, 2015)。

\section{4 声景生态学在生物多样性监测中的应用}

生物多样性评估作为保护生物学的基础和核 心内容(马克平, 2011), 常用于评估特定地区的生物 多样性、比较不同地区多样性组成的差异和监测生 物多样性的动态变化。传统的生物多样性评估方法, 如快速生物多样性评估和物种编目调查等, 受环境 条件、人力、物力的限制, 通常存在数据收集耗时
长、数据量贵乏、数据采集标准与数据质量参差不 齐等问题(Heywood \& Watson, 1995; David, 2005)。

基于“较高的物种丰富度可转化为较高的声学 复杂性, 假设, 声景生态学研究开发了一系列声学 指标提取音频中的信息指示生物多样性(Boelman et al, 2007; Sueur et al, 2008; Pieretti et al, 2011; Depraetere et al, 2012)。一方面, $\alpha$ 声学指标(测量声 学熵指数, 如 $H, A C I, A D I, A E I$ 等)可以评估某一特 定群落中的声音多样性。通过建立声学指标与实地 调查获得的物种信息, 或与从录音提取的声音类型 (比如将声音片段按频谱图相似性划分的若干类型, Desjonqueres et al, 2015)信息间的关系, 采用声学 指标评估发声群落的物种多样性。在陆地环境中, 声学指标已被用于评估昆虫群落物种丰富度(Roca \& Proulx, 2016)和鸟类群落物种丰富度、多度与组 成(Pieretti et al, 2011; Depraetere et al, 2012; Borker et al, 2014; Towsey et al, 2014), 如Gasc等(2013a)尝 试建立了声学指标与鸟类功能多样性、系统发育多 样性间的关系。另一方面, $\beta$ 声学指标可以度量不同 群落间声音多样性的差异。如, 声学差异性指数 $(D)$ 与频域差异性指数 $\left(D_{\mathrm{f}}\right)$ 均能探测到不同群落间物种 组成的区别(Sueur et al, 2008; Gasc et al, 2013b)。除 了用声学指标指示多样性外, 蒋锦刚等(2016)将遥 感领域常用的面向对象图像分割技术用于分割语 谱图, 试图用斑块统计分析方法提取音频中鸟类的 多样性信息。

Buxton等(2018b)对声学指标的荟萃分析结果 表明, 声学指标在大多数情况下 $(74 \%, n=151)$ 与生 物发声活动水平或生物多样性(如物种丰富度)显著 相关; 但同时还有部分指标(如 $\beta$ 声学指标，在 $50 \%$ 的情况下)并不能如实反映生物信息。本文作者统计 了12篇在陆地环境中研究声学指标与生物多样性 水平相关性的文献, 结果显示相关性系数绝对值在 0.01-0.97之间(图3)。在湖泊环境中, 声音丰富度指 数 $(A R)$ 与湖泊中声音类型的丰富度 $(r=0.2)$ 和多度 $(r=0.19)$ 呈显著正相关(Desjonqueres et al, 2015)。在 海洋环境中, Parks等(2014)建立了鲸类鸣声频率与 噪声补偿后的熵指数 (noise-compensated entropy index, $\left.H_{\mathrm{N}}\right)$ 的线性回归模型 $\left(R^{2}=0.225\right)$, 用于评价海 洋中的生物多样性和生境健康状况; Harris等(2016) 发现, 声音复杂度指数 $(A C I)$ 是评估温带珊瑚礁鱼 类多样性的有效指标, $A C I$ 与鱼类香农指数 $\left(H^{\prime}\right)$ 的相 


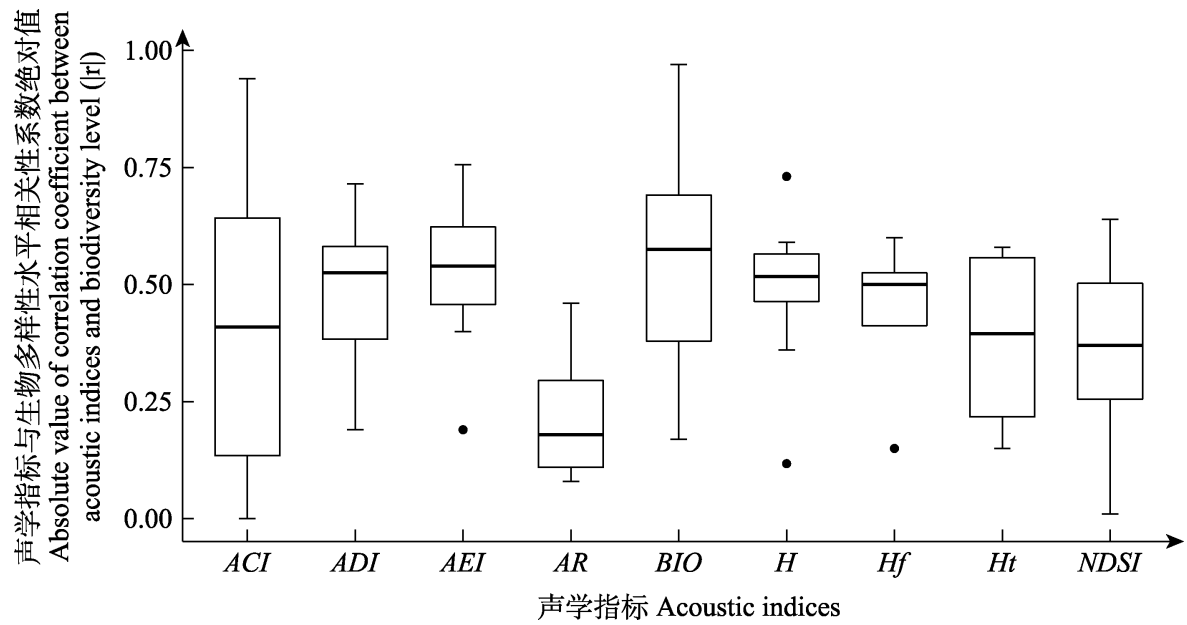

图3 声学指标与生物多样性水平相关性分析结果统计, $|\mathbf{r}|$ 为12篇陆生环境中研究声学指标与生物多样性耦合关系的相关性 系数绝对值。ACI: 声音复杂度指数; ADI: 声音多样性指数; AEI: 声音均匀度指数; AR: 声音丰富度指数; BIO: 生物声学指 数; $\boldsymbol{H}$ : 声学熵指数; $H_{f}$ : 频谱熵指数; $H_{\mathrm{t}}$ : 时间熵指数; NDSI: 标准化声景差异指数。

Fig. 3 Correlation between acoustic indices and biodiversity level, $|\mathrm{r}|$ is the absolute value of the correlation coefficient. ACI, Acoustic complexity index; ADI, Acoustic diversity index; AEI, Acoustic evenness index; AR, Acoustic richness index; BIO, Bioacoustic index; $H$, Acoustic entropy index; $H_{f}$, Spectral entropy index; $H_{\mathrm{t}}$, Temporal entropy index; $N D S I$, Normalised difference soundscape index.

关性系数达到 0.81 。整体而言, 声学指标与动物群 落多样性和发声活动水平之间关系的显著性, 在不 同研究区域、不同生态系统中并不相同，目前仍然 缺少能用于比较大尺度(比如中国跨生态区)生物多 样性差异的指标。

此外, 也有部分研究通过鸣声识别途径开展野 生动物个体、种群、群落水平的研究。例如, Chen 等(2020)利用深度学习算法开发了Waveman软件程 序, 可自动识别东南亚 36 种蝙蝠的叫声; Xia等 (2012)根据鸣唱的特征差异来区分北方中杜鹃 (Cuculus optatus)和喜马拉雅中杜鹃(C. saturates)。 对于个体间鸣声差异较大的物种，可以基于鸣声的 声学特征识别个体, 并估算种群数量。Gilbert等 (2002)通过鸣声识别大麻鳽(Botaurus stellaris)个体, 评估了英国境内大麻鳽繁殖种群大小及存活状况。 也有研究在自动识别物种鸣声的基础上计算声学 指数来估算种群大小。例如, Perez-Granados等(2019) 利用Song Scope 4.1.5 (Wildlife Acoustics 2011) 自动 辨识黄喉蜂虎 (Merops apiaster) 和杜氏百灵 (Chersophilus duponti), 并用发声活动频率指数 (vocal activity rate index)估算了两物种的种群大小。 物种识别途径仅被用于有限物种的研究, 当录音 内容涉及大量物种时, 识别出每个物种的难度和 时间成本大大增加, 监测整个群落的物种多样性
较为困难。

目前, 国内外已建立起若干区域性的声景监测 网络。如日本建立的Cyberforest森林声景监测网络, 该网络曾配合社交媒体开展森林鸟类普查(Saito et al, 2015); 中国台湾林业研究所及资讯科技创新研 究中心建立的亚洲声景监测网络(Asian Soundscape Monitoring Network); 美国为量化海洋声景基线水 平和评估动态变化趋势建立的国家公园管理局海 洋噪声参考站网络(National Park Service Ocean Noise Reference Station Network); 普渡大学为保护 自然声学遗址(natural soundscape heritage)建立的声 景生态学研究项目 (Soundscape Ecology Research Project, http://centerforglobalsoundscapes.org/pumiliosites); 中国科学院植物研究所自2014年起在钱江 源国家公园建立的声景监测平台。这些动态化的监 测平台为生物多样性和保护研究提供了有力数据 支撑。

\section{3 展望}

经过近十年的发展, 声景生态学研究已涉及陆 地、淡水和海洋生态系统中声景的时空格局、各组 分间的相互作用以及人类活动对声景的影响等多 个方面。自动录音技术的进步和声学指标的开发促 进了该学科的发展。同时, 声景生态学的理论和方 
法也已在调查生物多样性(Sueur et al, 2008)、评估 生态系统健康状况(Tucker et al, 2014)和保护地管理 成效(Borker et al, 2014)等方面得到初步应用。根据 该学科的发展态势, 我们提出以下几个今后应该重 点关注的方向。

\section{1 建设声学监测网络}

推进建立具有生态系统代表性的声学监测网 络, 收集长时期、大尺度的音频数据, 推动声景和 生物多样性、生态系统结构和功能的相互关系的研 究。声学监测网络的搭建需要开发低成本的录音设 备来满足用户大尺度研究的需求; 同时需要搭建数 据存储和管理平台，管理数据量庞大的录音文件。 也可以基于GIS技术开发声景信息系统(soundscape information systems, SIS), 便于研究人员在线查询、 分析和监听音频文件。由于网络平台日常维护成本 较高, 存储空间需求较大, 目前已建立的部分平台 难以长期运营, 如, 普渡大学开发的 Pumilio (http://pumilio.sourceforge.net)和密歇根州立大学开 发的REAL (http://real.msu.edu)。

\section{2 开发新方法和新指标}

鉴于许多现有声学指标的使用效果并不好 (Buxton et al, 2018b), 开发新方法和新指标, 从海 量的音频数据中提取有生态学意义的信息, 将是未 来声景生态学研究的一项关键内容。目前, 声景生 态学研究中调查的生态系统类型和动物类群仍有 限, 需要开展更多的野外研究, 以检验不同生境和 物种组合下声学指标评估生物多样性的效果及其 影响因素, 探讨不同声学指标的适用范围和局限, 寻求更为普适的方法和指标, 用于大尺度生物多样 性的研究和监测。同时, 在此过程中需建立声景标 准信息库, 收集声景各个组分(生物的、物理环境 的、人造的声音)的音频资料信息，提升单个项目对 声音信息的识别能力。

建立新的数据收集方法和分析方法, 从音频数 据中获取物种、个体层面更为精细的数据信息，可 以深度挖掘声景数据的信息。比如, 使用多台录音 机建立麦克风阵列, 定位、追踪声源具体位置, 研 究野生动物个体的行为(Payne et al, 2003)、个体间 的交互作用(Mennill \& Vehrencamp, 2008)、种群的 密度、对栖息地的利用等(Kirschel et al, 2011); 在自 动录音数据的基础上, 通过机器学习算法, 自动识 别录音中包含的鸟类(Aide et al, 2013; Stowell et al,
2019)、两栖爬行类(Ospina et al, 2013)、蝙蝠(Walters et $\mathrm{al}, 2012)$ 、鲸类(Mellinger et al, 2007)、昆虫 (Chesmore \& Ohya, 2004)等不同类群的物种, 甚至 分辨目标物种的不同个体(Terry et al, 2005), 获取 其性别、年龄(Thompson et al, 2010)等信息。当这些 新的或者正在被开发的方法应用到声景生态学研 究时, 可以拓宽和加深我们对音频数据的解读, 更 为准确地验证声学指标对生物多样性的指示作用, 促进更为有效的声学指标的开发。有必要强调的是, 作为一个跨学科的研究方向, 声景生态学需要生态 学和声学、计算机科学等不同领域研究者的合作, 仅生态学家开展此领域的研究会时时感到技术上 的困难。

声景监测获得的数据可与传统监测手段和其 他监测技术(比如，红外相机技术)所得数据相结合 (Bustamante et al, 2016; Campos-Cerqueira \& Aide, 2016)，用来评估多类群、多物种的空间分布格局， 以及识别保护优先区和制定土地利用规划(Rich et al，2019); 研究动物行为和种间关系, 识别生物多 样性对人为干扰或气候变化的响应(Buxton et al, 2018a)。由于从录音中提取的物种数据与红外相机 调查收集的物种数据类似，均为物种在调查点的分 布数据(presence数据), 在针对物种的研究中可借鉴 红外相机技术的数据分析方法, 比如, 采用占域模 型 (occupancy model) 估算物种栖息地占有率 (MacKenzie et al, 2002), 随机相遇模型 (random encounter model, REM)估算种群密度(Lucas et al, 2015), 贝叶斯层次模型 (Bayesian hierarchical modeling)评估物种丰富度(Tobler et al, 2015)等。

\section{3 记录和保存声景资源}

人类活动引起生态环境的改变和生物多样性 的丧失正在改变声景的组成。建议采集和保存不同 景观(尤其是原生自然景观)中的声景，服务于未来 的保护管理、成效评估和科学比较研究。人类社会 城镇化、人口增长、交通运输业和制造业的发展，使 原本丰富的自然声音逐渐被单调的人造声音所取 代。在此背景下，应在有代表性的生态系统内开展 系统的声学监测, 收集声学基线数据, 并通过长期 监测记录声景的变化。这样的长期声景监测数据, 可以记录下不断变迁的环境里自然、生物和人类活 动的信息，今日录制的音频可能成为来日的“声学 化石” (Pijanowski et al, 2011a)。尽管目前对音频数 
据的分析处理能力有限, 我们仍然可以收集和存储 录音数据, 构建典型群落的 “声学博物馆”, 如麦考 利博物馆(https://www.macaulaylibrary.org/)、Borror 生物声学实验室(https://blb.osu.edu/)、British Library Sounds (https://sounds.bl.uk/Environment/Soundscapes) 等。同时鼓励公众使用身边的移动录音设备采集声 景数据, 并做出描述与标注, 上传至数据库中共 享。随着分析技术的进步, 这些数据将在未来的研 究和自然教育中发挥作用。如Schafer (1994)所言, 我们应积极倾听自然中的声音, 认识其价值 (Murray, 1977), 从而进一步制定和实施有效的声景 保护政策。

同时，声景通过诸多途径为人类和野生动物带 来裨益和提供服务, 具有内禀价值, 应将其视作一 种资源进行保护和管理(Dumyahn \& Pijanowski, 2011), 通过保护自然环境的途径保护承载生物多 样性信息、文化价值和对人类健康有益的声景。人 类借由与声景的交互作用, 认知和理解周遭环境, 建构起“场所感(sense of place)”。研究表明, 场所内 的声景有助于提升个人对视觉景观的体验感 (Southworth, 1967)。健康的声环境对人类的生活和 野生动物的生存至关重要。噪声有损人体的精神和 身体 健康 (Staples，1996; Passchier-Vermeer \& Passchier, 2000), 影响野生动物对猎物的定位, 对 捕食者的趋避和种间的交流(Barber et al, 2010), 导 致野生动物的适合度下降(Reijnen et al, 1997; Stone, 2000)。因此，建议将栖息地声景的质量作为野生动 物栖息地保护与管理的一项内容, 保护自然原生景 观中的声环境, 促进声音信息在其中的有效传递。 此外，声景具有文化和历史价值，社会的加速发展 正在快速改变文化和历史遗迹中的声景(O’Connor, 2008)。在日本，声景被视为国家历史文化遗产的一 部分, 并被系统采集和记载(比如: 100 Soundscape of Japan: Preserving Our Heritage, Torigoe, 2003)。应 将自然中的健康声环境和对人类而言有历史文化 意义的声景视作一种资源进行保护，避免其遭受人 为开发和建设活动的破坏。

\section{参考文献}

Aide TM, Corrada-Bravo C, Campos-Cerqueira M, Milan C, Vega G, Alvarez R (2013) Real-time bioacoustics monitoring and automated species identification. PeerJ, 1, e103.

Au WWL, Mobley J, Burgess WC, Lammers MO, Nachtigall PE (2000) Seasonal and diurnal trends of chorusing humpback whales wintering in waters off western Maui. Marine Mammal Science, 16, 530-544.

Barber JR, Crooks KR, Fristrup KM (2010) The costs of chronic noise exposure for terrestrial organisms. Trends in Ecology \& Evolution, 25, 180-189.

Berg KS, Brumfield RT, Apanius V (2006) Phylogenetic and ecological determinants of the neotropical dawn chorus. Proceedings of the Royal Society B: Biological Sciences, 273, 999-1005.

Bittencourt L, Barbosa M, Secchi E, Lailson-Brito J, Azevedo A (2016) Acoustic habitat of an oceanic archipelago in the Southwestern Atlantic. Deep-Sea Research Part I: Oceanographic Research Papers, 115, 103-111.

Bobryk CW, Rega-Brodsky CC, Bardhan S, Farina A, He HS, Jose S (2016) A rapid soundscape analysis to quantify conservation benefits of temperate agroforestry systems using low-cost technology. Agroforestry Systems, 90, 997-1008.

Boelman NT, Asner GP, Hart PJ, Martin RE (2007) Multitrophic invasion resistance in Hawaii: Bioacoustics, field surveys, and airborne remote sensing. Ecological Applications, 17, 2137-2144.

Borker AL, McKown MW, Ackerman JT, Eagles-Smith CA, Tershy BR, Croll DA (2014) Vocal activity as a low cost and scalable index of seabird colony size. Conservation Biology, 28, 1100-1108.

Botteldooren D, Coensel BD, Muer TD (2006) The temporal structure of urban soundscapes. Journal of Sound and Vibration, 292, 105-123.

Brandes TS (2008) Automated sound recording and analysis techniques for bird surveys and conservation. Bird Conservation International, 18, S163-S173.

Brewer PG, Hester K (2009) Ocean acidification and the increasing transparency of the ocean to low-frequency sound. Oceanography, 22, 86-93.

Brown JL, Li SH, Bhagabati N (1999) Long-term trend toward earlier breeding in an American bird: A response to global warming? Proceedings of the National Academy of Sciences, USA, 96, 5565-5569.

Brumm H (2004) The impact of environmental noise on song amplitude in a territorial bird. Journal of Animal Ecology, 73, 434-440.

Burivalova Z, Towsey M, Boucher T, Truskinger A, Apelis C, Roe P, Game ET (2018) Using soundscapes to detect variable degrees of human influence on tropical forests in Papua New Guinea. Conservation Biology, 32, 205-215.

Burivalova Z, Wahyudi B, Boucher TM, Ellis P, Truskinger A, Towsey M, Roe P, Marthinus D, Griscom B, Game ET (2019) Using soundscapes to investigate homogenization of tropical forest diversity in selectively logged forests. Journal 
of Applied Ecology, 56, 2493-2504.

Bustamante MM, Roitman I, Aide TM, Alencar A, Anderson LO, Aragao L, Asner GP, Barlow J, Berenguer E, Chambers J, Costa MH, Fanin T, Ferreira LG, Ferreira J, Keller M, Magnusson WE, Morales-Barquero L, Morton D, Ometto JP, Palace M, Peres CA, Silverio D, Trumbore S, Vieira IC (2016) Toward an integrated monitoring framework to assess the effects of tropical forest degradation and recovery on carbon stocks and biodiversity. Global Change Biology, 22, 92-109.

Buxton RT, Brown E, Sharman L, Gabriele CM, McKenna MF (2016) Using bioacoustics to examine shifts in songbird phenology. Ecology and Evolution, 6, 4697-4710.

Buxton RT, Lendrum PE, Crooks KR, Wittemyer G (2018a) Pairing camera traps and acoustic recorders to monitor the ecological impact of human disturbance. Global Ecology and Conservation, 16, e00493.

Buxton RT, McKenna MF, Clapp M, Meyer E, Stabenau E, Angeloni LM, Crooks K, Wittemyer G (2018b) Efficacy of extracting indices from large-scale acoustic recordings to monitor biodiversity. Conservation Biology, 32, 1174-1184.

Cai XL, Liao WM, Zhang TH, Li XM, Chen FP, Deng RG (2010) Classification and evaluation of forest soundscape types. Acta Agriculturae Universitatis Jiangxiensis, 32, 1195-1201. (in Chinese with English abstract) [蔡学林, 廖 为明, 张天海, 李小毛, 陈飞平, 邓荣根 (2010) 森林声 景类型的划分与评价初探. 江西农业大学学报, 32, 1195-1201.]

Campos-Cerqueira M, Aide TM (2016) Improving distribution data of threatened species by combining acoustic monitoring and occupancy modelling. Methods in Ecology and Evolution, 7, 1340-1348.

Chen X, Zhao J, Chen YH, Zhou W, Hughes AC (2020) Automatic standardized processing and identification of tropical bat calls using deep learning approaches. Biological Conservation, 241, 108269.

Chesmore ED, Ohya E (2004) Automated identification of field-recorded songs of four British grasshoppers using bioacoustic signal recognition. Bulletin of Entomological Research, 94, 319-330.

Cuthill IC, Macdonald WA (1990) Experimental manipulation of the dawn and dusk chorus in the blackbird Turdus merula. Behavioral Ecology and Sociobiology, 26, 209-216.

Cynx J, Lewis R, Tavel B, Tse H (1998) Amplitude regulation of vocalizations in noise by a songbird, Taeniopygia guttata. Animal Behaviour, 56, 107-113.

David H (2005) Handbook of Biodiversity Methods: Survey, Evaluation and Monitoring. Cambridge University Press, Cambridge.

Deichmann JL, Hernandez-Serna A, Campos-Cerqueira M, Aide TM (2017) Soundscape analysis and acoustic monitoring document impacts of natural gas exploration on biodiversity in a tropical forest. Ecological Indicators, 74,
39-48.

Depraetere M, Pavoine S, Jiguet F, Gasc A, Duvail S, Sueur J (2012) Monitoring animal diversity using acoustic indices: Implementation in a temperate woodland. Ecological Indicators, 13, 46-54.

Desjonqueres C, Rybak F, Depraetere M, Gasc A, Le VI, Pavoine S, Sueur J (2015) First description of underwater acoustic diversity in three temperate ponds. PeerJ, 3, e1393.

Dooley JM, Brown MT (2019) The quantitative relation between ambient soundscapes and landscape development intensity in North Central Florida. Landscape Ecology, 35, 113-127.

Drafts B (2001) Acoustic wave technology sensors. IEEE Transactions on Microwave Theory and Techniques, 49, 795-802.

Dumyahn SL, Pijanowski BC (2011) Soundscape conservation. Landscape Ecology, 26, 1327-1344.

Ey E, Fischer J (2009) The “acoustic adaptation hypothesis"-A review of the evidence from birds, anurans and mammals. Bioacoustics, 19, 21-48.

Farina A, Gage SH (2017) Ecoacoustics: The Ecological Role of Sounds. Wiley, Hoboken.

Farina A, Pieretti N (2014) Sonic environment and vegetation structure: A methodological approach for a soundscape analysis of a Mediterranean maqui. Ecological Informatics, 21, 120-132.

Farina A, Pieretti N, Morganti N (2013) Acoustic patterns of an invasive species: The redbilled leiothrix (Leiothrix lutea Scopoli 1786) in a Mediterranean shrubland. Bioacoustics, 22, 175-194.

Feng AS, Schul J (2007) Sound processing in real-world environments. Hearing and Sound Communication in Amphibians, 28, 323-350.

Fenton MB, Bell GP (1979) Echolocation and feeding behaviour in four species of Myotis (Chiroptera). Canadian Journal of Zoology, 57, 1271-1277.

Ferrari MCO, McCormick MI, Meekan MG, Simpson SD, Nedelec SL, Chivers DP (2018) School is out on noisy reefs: The effect of boat noise on predator learning and survival of juvenile coral reef fishes. Proceedings of the Royal Society B: Biological Sciences, 285, 20180033.

Ferreira L, Oliveira EG, Lopes LC, Brito MR, Baumgarten J, Rodrigues FH, Sousa-Lima R (2018) What do insects, anurans, birds, and mammals have to say about soundscape indices in a tropical savanna. Journal of Ecoacoustics, 2, $1-17$.

Fuller S, Axel AC, Tucker D, Gage SH (2015) Connecting soundscape to landscape: Which acoustic index best describes landscape configuration? Ecological Indicators, 58, 207-215.

Gage SH, Axel AC (2014) Visualization of temporal change in soundscape power of a Michigan lake habitat over a 4-year period. Ecological Informatics, 21, 100-109. 
Garrido SA, Bertolelli L, Maria HA, Yebra AM, Munasinghe K (2020) The FrogPhone: A novel device for real-time frog call monitoring. Methods in Ecology and Evolution, 11, 222-228.

Gasc A, Gottesman BL, Francomano D, Jung J, Durham M, Mateljak J, Pijanowski BC (2018) Soundscapes reveal disturbance impacts: Biophonic response to wildfire in the Sonoran Desert Sky Islands. Landscape Ecology, 33, 1399-1415.

Gasc A, Pavoine S, Lellouch L, Grandcolas P, Sueur J (2015) Acoustic indices for biodiversity assessments: Analyses of bias based on simulated bird assemblages and recommendations for field surveys. Biological Conservation, 191, 306-312.

Gasc A, Sueur J, Jiguet F, Devictor V, Grandcolas P, Burrow C, Depraetere M, Pavoine S (2013a) Assessing biodiversity with sound: Do acoustic diversity indices reflect phylogenetic and functional diversities of bird communities? Ecological Indicators, 25, 279-287.

Gasc A, Sueur J, Pavoine S, Pellens R, Grandcolas P (2013b) Biodiversity sampling using a global acoustic approach: Contrasting sites with microendemics in New Caledonia. PLoS ONE, 8, e65311.

Gibbs JP, Breisch AR (2001) Climate warming and calling phenology of frogs near Ithaca, New York, 1900-1999. Conservation Biology, 15, 1175-1178.

Gilbert G, Tyler GA, Smith KW (2002) Local annual survival of booming male great Bittern Botaurus stellaris in Britain, in the period 1990-1999. Ibis, 144, 51-61.

Gomez WE, Isaza CV, Daza JM (2018) Identifying disturbed habitats: A new method from acoustic indices. Ecological Informatics, 45, 16-25.

Greenfield MD (1994) Cooperation and conflict in the evolution of signal interactions. Annual Review of Ecology and Systematics, 25, 97-126.

Guan S, Vignola JF, Lin TH, Chou L (2016) Soundscape Characteristics of the Eastern Taiwan Strait Indo-Pacific Humpback Dolphin Habitat. https://ieeexplore-ieee-org-443. webvpn.las.ac.cn/document/7890674. (accessed on 2020-0827)

Hage SR, Jiang T, Berquist SW, Feng J, Metzner W (2013) Ambient noise induces independent shifts in call frequency and amplitude within the Lombard effect in echolocating bats. Proceedings of the National Academy of Sciences, USA, 110, 4063-4068.

Halfwerk W, Slabbekoorn H (2009) A behavioural mechanism explaining noise-dependent frequency use in urban birdsong. Animal Behaviour, 78, 1301-1307.

Hardouin LA, Robert D, Bretagnolle V (2008) A dusk chorus effect in a nocturnal bird: Support for mate and rival assessment functions. Behavioral Ecology and Sociobiology, 62, 1909-1918.

Harris SA, Shears NT, Radford CA (2016) Ecoacoustic indices as proxies for biodiversity on temperate reefs. Methods in Ecology and Evolution, 7, 713-724.

Heywood VH, Watson RT (1995) Global Biodiversity Assessment. Cambridge University Press, Cambridge.

Hill AP, Prince P, Piña CE, Doncaster CP, Snaddon JL, Rogers A (2018) AudioMoth: Evaluation of a smart open acoustic device for monitoring biodiversity and the environment. Methods in Ecology and Evolution, 9, 1199-1211.

Holles S, Simpson SD, Radford AN, Berten L, Lecchini D (2013) Boat noise disrupts orientation behaviour in a coral reef fish. Marine Ecology Progress Series, 485, 295-300.

Hutchinson JMC (2002) Two explanations of the dawn chorus compared: How monotonically changing light levels favour a short break from singing. Animal Behaviour, 64, 527-539.

Jiang JG, Shao XY, Wan HB, Qi JG, Jing CW, Cheng TY (2016) Bird diversity research using audio record files and the spectrogram segmentation method. Acta Ecologica Sinica, 36, 7713-7723. (in Chinese with English abstract) [蒋锦刚, 邵小云, 万海波, 齐家国, 荆长伟, 程天佑 (2016) 基于语谱图特征信息分割提取的声景中鸟类生物 多样性分析. 生态学报, 36, 7713-7723.]

Jiang T, Guo X, Lin A, Wu H, Sun C, Feng J, Kanwal JS (2019) Bats increase vocal amplitude and decrease vocal complexity to mitigate noise interference during social communication. Animal Cognition, 22, 199-212.

Joo W, Gage SH, Kasten EP (2011) Analysis and interpretation of variability in soundscapes along an urban-rural gradient. Landscape and Urban Planning, 103, 259-276.

Kasten EP, Gage SH, Fox J, Joo W (2012) The remote environmental assessment laboratory's acoustic library: An archive for studying soundscape ecology. Ecological Informatics, 12, 50-67.

Kirschel ANG, Cody ML, Harlow ZT, Promponas VJ, Vallejo EE, Taylor CE (2011) Territorial dynamics of Mexican ant-thrushes Formicarius moniliger revealed by individual recognition of their songs. Ibis, 153, 255-268.

Krause BL (1987) Bioacoustics, habitat ambience in ecological balance. Whole Earth Review, 57, 14-18.

Krause BL (1993) The niche hypothesis: A virtual symphony of animal sounds, the origins of musical expression and the health of habitats. The Soundscape Newsletter, 6, 6-10.

Krause BL, Farina A (2016) Using ecoacoustic methods to survey the impacts of climate change on biodiversity. Biological Conservation, 195, 245-254.

Krause BL, Gage SH, Joo W (2011) Measuring and interpreting the temporal variability in the soundscape at four places in Sequoia National Park. Landscape Ecology, 26, 1247-1256.

Kuehne LM, Padgham BL, Olden JD (2013) The soundscapes of lakes across an urbanization gradient. PLoS ONE, 8, e55661.

Lecchini D, Bertucci F, Gache C, Khalife A, Besson M, Roux N, Berthe C, Singh S, Parmentier E, Nugues MM (2018) 
Boat noise prevents soundscape-based habitat selection by coral planulae. Scientific Reports, 8, 9283.

Lengagne T, Aubin T, Lauga J, Jouventin P (1999) How do king penguins (Aptenodytes patagonicus) apply the mathematical theory of information to communicate in windy conditions? Proceedings of the Royal Society B: Biological Sciences, 266, 1623-1628.

Lillis A, Bohnenstiehl D, Peters JW, Eggleston D (2016) Variation in habitat soundscape characteristics influences settlement of a reef-building coral. PeerJ, 4, e2557.

Lillis A, Eggleston DB, Bohnenstiehl DR (2014) Soundscape variation from a larval perspective: The case for habitat-associated sound as a settlement cue for weakly swimming estuarine larvae. Marine Ecology Progress Series, 509, 57-70.

Lillis A, Mooney TA (2018) Snapping shrimp sound production patterns on Caribbean coral reefs: Relationships with celestial cycles and environmental variables. Coral Reefs, 37, 597-607.

Lin T, Tsao Y, Wang Y, Yen H, Lu S (2017) Computing biodiversity change via a soundscape monitoring network. 2017 Pacific Neighborhood Consortium Annual Conference and Joint Meetings (PNC), IEEE, 128-133.

Liu J, Kang J, Luo T, Behm H, Coppack T (2013) Spatiotemporal variability of soundscapes in a multiple functional urban area. Landscape and Urban Planning, 115, $1-9$.

Lombard E (1911) Le signe de l'élévation de la voix. Annales des Maladies de L'Oreille et du Larynx, 37, 101-119.

Lucas TCD, Moorcroft EA, Freeman R, Rowcliffe JM, Jones KE (2015) A generalised random encounter model for estimating animal density with remote sensor data. Methods in Ecology and Evolution, 6, 500-509.

Luo J, Goerlitz HR, Brumm H, Wiegrebe L (2015a) Linking the sender to the receiver: Vocal adjustments by bats to maintain signal detection in noise. Scientific Reports, 5, 18556.

Luo J, Siemers BM, Koselj K (2015b) How anthropogenic noise affects foraging. Global Change Biology, 21, 3278-3289.

Luo JH, Koselj K, Zsebok S, Siemers BM, Goerlitz HR (2014) Global warming alters sound transmission: Differential impact on the prey detection ability of echolocating bats. Journal of the Royal Society Interface, 11, 20130961.

Lü J, Guo Q, Feng HN (2012) Anomalous infrasonic waves before an small earthquake in Beijing. Chinese Journal of Geophysics, 10, 3379-3385. (in Chinese with English abstract) [吕君, 郭泉, 冯浩楠 (2012) 北京地震前的异常 次声波. 地球物理学报, 10, 3379-3385.]

Ma KP (2011) Assessing progress of biodiversity conservation with monitoring approach. Biodiversity Science, 19, 125-126. (in Chinese) [马克平 (2011) 监测是评估生物多 样性保护进展的有效途径. 生物多样性, 19, 125-126.]
MacKenzie DI, Nichols JD, Lachman GB, Droege S, Royle JA, Langtimm CA (2002) Estimating site occupancy rates when detection probabilities are less than one. Ecology, 83, 2248-2255.

Martin SB, Popper AN (2016) Short- and long-term monitoring of underwater sound levels in the Hudson River (New York, USA). Journal of the Acoustical Society of America, 139, 1886-1897.

Mellinger DK, Stafford KM, Moore SE, Dziak RP, Matsumoto $H$ (2007) An overview of fixed passive acoustic observation methods for cetaceans. Oceanography, 20, 36-45.

Mennill DJ, Vehrencamp SL (2008) Context-dependent functions of avian duets revealed by microphone-array recordings and multispeaker playback. Current Biology, 18, 1314-1319.

Morton ES (1975) Ecological sources of selection on avian sounds. The American Naturalist, 109, 17-34.

Murray SR (1977) The Soundscape: The Tuning of the World. Knopf, New York.

O'Connor P (2008) The sound of silence: Valuing acoustics in heritage conservation. Geographical Research, 46, 361-373.

Ospina OE, Villanueva-Rivera LJ, Corrada-Bravo CJ, Aide TM (2013) Variable response of anuran calling activity to daily precipitation and temperature: Implications for climate change. Ecosphere, 4, 1-12.

Parks SE, Miksis-Olds JL, Denes SL (2014) Assessing marine ecosystem acoustic diversity across ocean basins. Ecological Informatics, 21, 81-88.

Parmesan C (2006) Ecological and evolutionary responses to recent climate change. Annual Review of Ecology, Evolution, and Systematics, 37, 637-669.

Parsons MJG, Salgado-Kent CP, Marley SA, Gavrilov AN, McCauley RD (2016) Characterizing diversity and variation in fish choruses in Darwin Harbour. Ices Journal of Marine Science, 73, 2058-2074.

Passchier-Vermeer W, Passchier WF (2000) Noise exposure and public health. Environmental Health Perspectives, 108, 123-131.

Payne KB, Langbauer WR, Thomas EM (1986) Infrasonic calls of the Asian elephant (Elephas maximus). Behavioral Ecology and Sociobiology, 18, 297-301.

Payne KB, Thompson M, Kramer L (2003) Elephant calling patterns as indicators of group size and composition: The basis for an acoustic monitoring system. African Journal of Ecology, 41, 99-107.

Pekin BK, Jung J, Villanueva-Rivera LJ, Pijanowski BC, Ahumada JA (2012) Modeling acoustic diversity using soundscape recordings and LIDAR-derived metrics of vertical forest structure in a neotropical rainforest. Landscape Ecology, 27, 1513-1522.

Perez-Granados C, Bota G, Giralt D, Barrero A, Gomez-Catasus J, Rosa DBL, Traba J (2019) Vocal activity rate index: A useful method to infer terrestrial bird 
abundance with acoustic monitoring. Ibis, 161, 901-907.

Pieretti N, Farina A, Morri D (2011) A new methodology to infer the singing activity of an avian community: The acoustic complexity index (ACI). Ecological Indicators, 11, 868-873.

Pijanowski BC, Farina A, Gage SH, Dumyahn SL, Krause BL (2011a) What is soundscape ecology? An introduction and overview of an emerging new science. Landscape Ecology, 26, 1213-1232.

Pijanowski BC, Villanueva-Rivera LJ, Dumyahn SL, Farina A, Krause BL, Napoletano BM, Gage SH, Pieretti N (2011b) Soundscape ecology: The science of sound in the landscape. BioScience, 61, 203-216.

Putland RL, Constantine R, Radford CA (2017) Exploring spatial and temporal trends in the soundscape of an ecologically significant embayment. Scientific Reports, 7, 5713.

Radford CA, Jeffs AG, Tindle CT, Montgomery JC (2008) Temporal patterns in ambient noise of biological origin from a shallow water temperate reef. Oecologia, 156, 921-929.

Reijnen R, Foppen R, Veenbaas G (1997) Disturbance by traffic of breeding birds: Evaluation of the effect and considerations in planning and managing road corridors. Biodiversity \& Conservation, 6, 567-581.

Rice AN, Soldevilla MS, Quinlan JA (2017) Nocturnal patterns in fish chorusing off the coasts of Georgia and eastern Florida. Bulletin of Marine Science, 93, 455-474.

Rich LN, Furnas BJ, Newton DS, Brashares JS (2019) Acoustic and camera surveys inform models of current and future vertebrate distributions in a changing desert ecosystem. Diversity and Distributions, 25, 1441-1456.

Roca IT, Proulx R (2016) Acoustic assessment of species richness and assembly rules in ensiferan communities from temperate ecosystems. Ecology, 97, 116-123.

Rodriguez A, Gasc A, Pavoine S, Grandcolas P, Gaucher P, Sueur J (2014) Temporal and spatial variability of animal sound within a neotropical forest. Ecological Informatics, 21, 133-143.

Ruppé L, Clément G, Herrel A, Ballesta L, Décamps T, Kéver L, Parmentier E (2015) Environmental constraints drive the partitioning of the soundscape in fishes. Proceedings of the National Academy of Sciences, USA, 112, 6092-6097.

Russ J (1999) The Bats of Britain and Ireland: Echolocation Calls, Sound Analysis and Species Identification. Alan Books, Powys.

Saito K, Nakamura K, Ueta M, Kurosawa R, Fujiwara A, Kobayashi HH, Nakayama M, Toko A, Nagahama K (2015) Utilizing the Cyberforest live sound system with social media to remotely conduct woodland bird censuses in Central Japan. Ambio, 44, 572-583.

Schafer RM (1969) The New Soundscape. Berandol Music Limited, Toronto.

Schafer RM (1970) The Book of Noise. Price Print, Vancouver.
Schafer RM (1994) The Soundscape: Our Sonic Environment and the Tuning of the World. Destiny Books, Rochester.

Sethi SS, Ewers RM, Jones NS, Orme CDL, Picinali L (2018) Robust, real-time and autonomous monitoring of ecosystems with an open, low-cost, networked device. Methods in Ecology and Evolution, 12, 2383-2387.

Sierro J, Schloesing E, Pavon I, Gil D (2017) European blackbirds exposed to aircraft noise advance their chorus, modify their song and spend more time singing. Frontiers in Ecology and Evolution, 5, 1-13.

Slabbekoorn H, Peet M (2003) Ecology: Birds sing at a higher pitch in urban noise. Nature, 424, 267.

Smott S, Monczak A, Miller ME, Montie EW (2018) Boat noise in an estuarine soundscape-A potential risk on the acoustic communication and reproduction of soniferous fish in the May River, South Carolina. Marine Pollution Bulletin, 133, 246-260.

Snell-Rood EC (2012) The effect of climate on acoustic signals: Does atmospheric sound absorption matter for bird song and bat echolocation? Journal of the Acoustical Society of America, 131, 1650-1658.

Southworth MF (1967) The Sonic Environment of Cities. PhD dissertation, Massachusetts Institute of Technology, Cambridge.

Staaterman E, Paris CB, DeFerrari HA, Mann DA, Rice AN, D’Alessandro EK (2014) Celestial patterns in marine soundscapes. Marine Ecology Progress Series, 508, 17-32.

Staples SL (1996) Human response to environmental noise: Psychological research and public policy. American Psychologist, 51, 143-150.

Stone E (2000) Separating the noise from the noise: A finding in support of the "niche hypothesis," that birds are influenced by human-induced noise in natural habitats. Anthrozoös, 13, 225-231.

Stowell D, Wood MD, Pamuta H, Stylianou Y, Glotin H (2019) Automatic acoustic detection of birds through deep learning: The first bird audio detection challenge. Methods in Ecology and Evolution, 10, 368-380.

Sueur J, Farina A, Gasc A, Pieretti N, Pavoine S (2014) Acoustic indices for biodiversity assessment and landscape investigation. Acta Acustica, 100, 772-781.

Sueur J, Pavoine S, Hamerlynck O, Duvail S (2008) Rapid acoustic survey for biodiversity appraisal. PLoS ONE, 3, e4065.

Sugai LSM, Silva TSF, Ribeiro JJW, Llusia D (2019) Terrestrial passive acoustic monitoring: Review and perspectives. BioScience, 69, 15-25.

Swiston KA, Mennill DJ (2009) Comparison of manual and automated methods for identifying target sounds in audio recordings of Pileated, Pale-billed, and putative Ivory-billed woodpeckers. Journal of Field Ornithology, 80, 42-50.

Terry AMR, Peake TM, McGregor PK (2005) The role of vocal 
individuality in conservation. Frontiers in Zoology, 2, 10.

Thompson EA (2004) The Soundscape of Modernity: Architectural Acoustics and the Culture of Listening in America. MIT Press, Cambridge.

Thompson ME, Schwager SJ, Payne KB (2010) Heard but not seen: An acoustic survey of the African forest elephant population at Kakum Conservation Area, Ghana. African Journal of Ecology, 48, 224-231.

Tobler MW, Zúñiga HA, Carrillo-Percastegui SE, Powell GVN, Lukacs P (2015) Spatiotemporal hierarchical modelling of species richness and occupancy using camera trap data. Journal of Applied Ecology, 52, 413-421.

Torigoe K (2003) Insights taken from three visited soundscapes in Japan. The Journal of Acoustic Ecology, 6, 9-12.

Towsey M, Planitz B, Nantes A, Wimmer J, Roe P (2012) A toolbox for animal call recognition. Bioacoustics, 21, 107-125.

Towsey M, Wimmer J, Williamson I, Roe P (2014) The use of acoustic indices to determine avian species richness in audio-recordings of the environment. Ecological Informatics, 21, 110-119.

Truax B (2008) Soundscape composition as global music: Electroacoustic music as soundscape. Organised Sound, 13, 103-109.

Tucker D, Gage SH, Williamson I, Fuller S (2014) Linking ecological condition and the soundscape in fragmented Australian forests. Landscape Ecology, 29, 745-758.

Velásquez NA, Moreno-Gómez FN, Brunetti E, Penna M (2018) The acoustic adaptation hypothesis in a widely distributed South American frog: Southernmost signals propagate better. Scientific Reports, 8, 6990.

Venter PJ, Hanekom JJ (2010) Automatic detection of African elephant (Loxodonta africana) infrasonic vocalisations from recordings. Biosystems Engineering, 106, 286-294.

Villanueva-Rivera LJ (2014) Eleutherodactylus frogs show frequency but no temporal partitioning: Implications for the acoustic niche hypothesis. PeerJ, 2, e496.

Villanueva-Rivera LJ, Pijanowski BC, Doucette J, Pekin B (2011) A primer of acoustic analysis for landscape ecologists. Landscape Ecology, 26, 1233-1246.

Walters CL, Freeman R, Collen A, Dietz C, Fenton MB, Jones G, Obrist MK, Puechmaille SJ, Sattler T, Siemers BM, Parsons S, Jones KE (2012) A continental-scale tool for acoustic identification of European bats. Journal of Applied Ecology, 49, 1064-1074.

Whytock RC, Christie J (2017) Solo: An open source, customizable and inexpensive audio recorder for bioacoustic research. Methods in Ecology and Evolution, 8, 308-312.

Wimmer J, Towsey M, Roe P, Williamson I (2013) Sampling environmental acoustic recordings to determine bird species richness. Ecological Applications, 23, 1419-1428.

Xia C, Lin X, Liu W, Lloyd H, Zhang Y (2012) Acoustic identification of individuals within large avian populations: A case study of the brownish-flanked bush warbler, South-Central China. PLoS ONE, 7, e42528.

Zhao Z, Xu ZY, Bellisario K, Zeng RW, Li N, Zhou WY, Pijanowski BC (2019) How well do acoustic indices measure biodiversity? Computational experiments to determine effect of sound unit shape, vocalization intensity, and frequency of vocalization occurrence on performance of acoustic indices. Ecological Indicators, 107, 105588.

(责任编委: 丁平 责任编辑: 问文杰) 